\title{
The effects of a multicomponent dyadic intervention on the mood, behavior, and physical health of people with dementia: a randomized controlled trial
}

\author{
Anna-Eva Prick' \\ Jacomine de Lange ${ }^{2}$ \\ Erik Scherder ${ }^{3}$ \\ Jos Twisk ${ }^{4}$ \\ Anne Margriet Pot ${ }^{1,5,6}$ \\ 'Department of Clinical Psychology \\ and the $\mathrm{EMGO}^{+}$Institute for \\ Health and Care Research, Faculty \\ of Psychology and Education, \\ VU University, Amsterdam, the \\ Netherlands; ${ }^{2}$ Research Centre \\ Innovations in Care, Rotterdam \\ University of Applied Sciences, \\ Rotterdam, the Netherlands; \\ ${ }^{3}$ Department of Clinical \\ Neuropsychology, Faculty of \\ Psychology and Education, \\ VU University, Amsterdam, the \\ Netherlands; ${ }^{4}$ Department of \\ Epidemiology and Biostatistics and \\ the $\mathrm{EMGO}^{+}$Institute for Health and \\ Care Research, VU University Medical \\ Center, Amsterdam, the Netherlands; \\ ${ }^{5}$ Program on Ageing, Institute \\ of Mental Health and Addiction, \\ Utrecht, the Netherlands; ${ }^{6}$ School of \\ Psychology, University of Queensland, \\ Brisbane, QLD, Australia
}

This article was published in the following Dove Press journal:

Clinical Interventions in Aging

31 March 2016

Number of times this article has been viewed

Purpose: The effects of a multicomponent dyadic intervention on the mood, behavior, and physical health of people with dementia living in the community were evaluated in a randomized controlled trial. This multicomponent dyadic intervention is a translated and adapted version of an intervention that has been shown to be effective for people with dementia in the US.

Patients and methods: People with dementia living in the community and their family caregivers ( $\mathrm{N}=111$ caregiver-care recipient dyads) were randomly assigned to the intervention and comparison group. The intervention group received home-based physical exercise training, psycho-education, communication skills training, and pleasant activities training during 3 months directed at both the person with dementia and the caregiver. Mood, behavior, and physical health were measured at baseline, 3 months, and 6 months. The effects of the study were determined by using generalized estimating equations based on an intention-to-treat analysis.

Results: Analyses showed no beneficial effects over time on any of the outcome measures.

Conclusion: This study showed no effects. The negative results in this study compared to the study that has been carried out in the US might be explained by the translation, adaptation, and shortening of the intervention used in the US, and a different social context. In addition, the results might be explained by the lack of room for improvement and by experiencing the intervention as too much of a burden. Furthermore, improving physical health might only be effective if the physical exercises are of moderate-to-high-intensity and are tailored in accordance with participants' preferences and needs. For future studies, because dyads often commented positively about the pleasure and support they received, it might also be valuable to measure quality of life outcomes such as relationship quality, pleasure, and self-esteem in dyadic focused interventions.

Keywords: intervention, prevention, mental health, dementia, caregivers

\section{Introduction}

Dementia is a serious disease for older people, which affects cognitive functioning, behavioral functioning, mood, and physical health. ${ }^{1-3}$ The problems increase during the course of the disease resulting in not being fully capable of self-care anymore. As a consequence, the quality of life of people with dementia may decrease, and caregiver distress increases as well as the probability of institutionalization. ${ }^{4}$ Since most people with dementia will continue to live at home with the help of a family caregiver as long as possible, effective community-based interventions that stabilize or delay the development of dementia-related problems are highly relevant.

Psychosocial interventions have the potential to improve the mood, behavioral and physical health problems of people with dementia. ${ }^{5-7}$ More specific, psychosocial interventions that include a combination of intervention strategies (multicomponent 
intervention) addressing both the person with dementia and their caregiver (dyadic or combined interventions) are effective for both the caregiver and the person with dementia. ${ }^{5,6,8,9}$ Multicomponent dyadic interventions comprise components such as information, training for activities of daily life, walking or exercise, and environmental adaptations for the person with dementia; and information, psycho-education, skills training, and coping strategies for the caregiver. Combining intervention types for both people with dementia and their caregivers may have possible additional effects, with each intervention augmenting the effect of the other. Addressing the dyad is seen as effective because of the mutual influence between the person with dementia and the caregiver. For example, behavioral problems in people with dementia may increase the caregiver's burden; caregiver support will influence both the mood of the caregiver and the behavior of the person with dementia. ${ }^{10}$ The authors of a recent review studying the effects of dyadic psychosocial programs found that 19 of the 20 included programs showed significant effects on the person with dementia, the caregiver, or both. ${ }^{9}$ When focusing on the effects on the person with dementia, the review showed strong evidence for the outcome of mood and moderate evidence for the outcome of behavioral problems. Furthermore, the review showed that programs that target competence of the caregiver and behavioral problems and/or activity for daily life dependency of the person with dementia seem to be promising. Especially, active training for activities and communication skills improves results for both members of the dyad.

The intervention study of Teri et al combined exercises with behavioral management techniques and pleasant activities training directed at both the person with dementia and their caregiver, which showed positive effects on the mood and physical functioning of people with dementia. ${ }^{11}$ The effects on caregivers' health were not studied, although, performing pleasant activities and physical exercises by caregivers themselves have been found to improve caregivers' well-being in earlier research. ${ }^{12,13}$ Furthermore, earlier research showed that the mood of caregivers is related to the mood of the care receiver. ${ }^{14,15}$ In addition, since physical exercise in combination with behavioral activation and pleasant activities seems to be promising and can easily be provided at home for people with dementia, an intervention program like the one studied by Teri et al might have opportunities in other countries outside the US as well. From an efficiency point of view, it is worthwhile to translate an intervention program already developed and found to be effective in another country. ${ }^{11}$ After a pilot study, using a translated version of the Teri intervention, we made changes to the original intervention components and duration to adapt the intervention to the Dutch care situation. ${ }^{11,16}$

The purpose of the present randomized controlled trial (RCT) was to investigate the effects of this multicomponent dyadic intervention (an adjusted and elaborated version of the intervention of Teri et al) on the mood, behavior, and physical health of people with dementia living at home in the Netherlands; the effects on caregivers are published elsewhere. ${ }^{11,17,18}$

\section{Methods \\ Design}

Trial registration: Dutch trial register: NTR1802, registration date May 6, 2009. An RCT design with three measurements (baseline, 3 months post-measurement, and 6 month follow-up measurement) was used. Informed consent was signed by both the caregiver and the person with dementia individually before the start of the study. After baseline assessment, a total of 111 dyads were randomly assigned to the intervention $(n=57)$ or comparison group $(n=54)$ with a minimal intervention.

The study design and a process evaluation of this RCT has been described extensively elsewhere. ${ }^{16,17}$

\section{Procedure}

An independent researcher made the random allocation schedule (in blocks of 20 dyads), using Random Allocation Software (Version 1). Dyads and coaches were aware of the treatment assigned. At the start of each measurement, the examiners were blinded to the group allocation and dyads were asked not to disclose their group allocation.

\section{Ethical aspects}

The Medical Ethics Committee of the VU University Medical Center approved the study protocol (registration number 2008/320).

\section{Study population}

Via Alzheimer Cafes (easily accessible meetings for people with dementia, their caregivers, and others), case managers, flyers, advertisements in newspapers, and the Internet 146 persons with dementia and their caregivers were recruited between November 2008 and June 2012 throughout the Netherlands.

Inclusion criteria for people with dementia were a diagnosis of dementia made by a physician (for instance a general practitioner, psychiatrist, geriatrician or a neurologist), 
minimum age 55 years, and living at home with a caregiver willing to participate in the training sessions. Exclusion criteria were the use of antidepressants, the presence of psychotic symptoms, Mini Mental State Examination (MMSE) score $<14$, and receiving more than 2 days respite care in a day care facility.

Family caregivers were defined as spouses or adult relatives who live with or spend a minimum of 4 hours every day with the person with dementia. Caregivers needed to have at least some depressive symptoms (Centre for Epidemiologic Studies-Depression score $>5$ ) to be included in the study. Caregivers were excluded in the case of physical disorders that hamper assistance with the exercises, presence of psychotic symptoms, and use of antidepressants.

\section{Intervention}

The intervention was largely based on an intervention of Teri et al which combined a physical exercise program (30 minutes of daily moderate-to-high-intensity exercise) for people with dementia with teaching caregivers how to manage behavioral problems (Activating events Beliefs Consequences [ABC] training) and to identify pleasant activities. ${ }^{11,19,20}$ After a pilot study, using a translated version of the Teri et al intervention, we made changes to the original intervention components and duration (eight instead of the original 12 sessions) to adapt the intervention to the Dutch care situation. ${ }^{16}$ Because the evaluation of the pilot study showed that a proper execution of the intervention took more than 1 hour, we decided to concentrate on physical exercise, education, and pleasant activities training and to omit the time-consuming $\mathrm{ABC}$ training. This choice was further motivated by the fact that cognitive reframing (based on the ABC theory) has already been shown to be effective, whereas the effectiveness of physical exercise and pleasant activities training for people with dementia and their caregivers in the community is less well-studied. ${ }^{21}$ We decided to pay much more attention to the pleasant activity training with the underlying idea that physical activity could also be a pleasant activity: we performed the pleasant activity training in several sessions (session 3-8) instead of one session, and we taught caregivers not only to plan pleasant activities for people with dementia (planned together with the person with dementia as much as possible), but also for caregivers themselves and together with the person with dementia present).

In the final adapted intervention, dyads allocated to the intervention group received a multicomponent dyadic intervention consisting of physical exercise training, psychoeducation, communication skills training, and pleasant activities training. A personal coach who visited the dyads in their own homes for eight 1-hour-long sessions during 3 months delivered this multicomponent dyadic intervention. During the first month, the coach visited the dyads weekly followed by biweekly sessions over the next 8 weeks. During the physical exercise training, four types of exercises (flexibility, strengthening, balance, and endurance) were gradually taught and practiced with the intention to motivate dyads to complete 30 minutes of active exercise at least 3 days a week. Each exercise training session started with careful stretching serving as "warm-up" for the other exercises, and increasing participants' awareness of their muscles. All exercises focused on ease, availability, and pleasantness. ${ }^{22}$

The goal of psycho-education was to educate the dyad about dementia and its impact on the person with dementia and caregiver, and how to deal with it. In the communication skills training, the coach discussed techniques for facilitating dyad's communication. Pleasant activities training stimulated the planning of pleasant activities in daily life for both individual members of the dyad and for them together as a dyad to reduce psychological distress. Alongside the instruction visits, all dyads received a user manual with pictures of the exercises and easy-to-read instructions including sessionspecific worksheets with specific information and psychoeducation for each visit.

\section{Comparison group with minimal intervention}

Participants who were assigned to the comparison group received a minimal intervention in addition to usual care. The minimal intervention consisted of written information monthly bulletins sent to the dyads with general information about dementia such as information about driving a car and general health (three in total), and monthly phone calls from one of the coaches (three in total). The goal of the maximal 10 minute-phone call was emotional support by listening.

\section{Measures \\ Baseline variables}

To collect general and demographic information, questions were asked concerning age, sex, birth date, education, dementia type, comorbidity, medication use, cognitive functioning, and the client-caregiver relationship. The education level was determined by a seven-point scale varying from less than elementary school (0) to technical college and university (6) ${ }^{23}$ In people with dementia, the global level of cognitive functioning was determined using the Dutch version of the MMSE and the Global Deterioration Scale (GDS) to clinically distinguish between the global stages from normality to severe 
dementia. ${ }^{24-26}$ Medication use was coded according to the Anatomical Therapeutic Chemical Classification System, an international standard for drug utilization studies. Comorbid conditions were ascertained from the caregiver and categorized according to the main categories based on the International Statistical Classification of Diseases. Furthermore, the status of apolipoprotein $\varepsilon 4$ allele (APOE- $\varepsilon 4$ ) was determined as a baseline variable. APOE- $\varepsilon 4$ carriers show an increased risk of cognitive decline and, thereby, may show a greater response to physical activity than non-carriers. ${ }^{27,28}$ To control for the possible influence of APOE- $\varepsilon 4$ on the treatment effects, we analyzed APOE- $\varepsilon 4$. Buccal swabs were taken by using Catch-all collection swabs (Epicentre, Madison, WI, USA) and DNA was isolated from the swab. ${ }^{29}$ ApoE genotype was indicated as APOE- $\varepsilon 4$ present or absent.

\section{Primary outcomes}

We mainly used the same scales as used by Teri et al ${ }^{11}$ to enable a comparison of the results of both studies. In line with Teri et $\mathrm{al}^{11}$ physical health was measured by two subscales (physical functioning and physical role functioning) of the Medical Outcome Study 36-item Short-Form Health Survey (SF-36) and three subscales (mobility range and mobility control) from the Sickness Impact Profile (SIP). ${ }^{30-33}$ In addition to Teri et al, ${ }^{11}$ we measured depression using clients' self-report with the Geriatric Depression Scale 15 (GDS-15) in addition to two observation scales: the Cornell Scale for Depression in Dementia (Cornell) and the Depression Rating Scale (DRS) of the Resident Assessment Instrument Home Care (RAI-HC) (DRS-RAI-HC). ${ }^{34-40}$

To measure the level of depression of people with dementia, the caregiver together with the interviewer completed the Dutch version of the 19-item Cornell. ${ }^{36,37}$ The scale has 19 items that are based on the week prior to the assessment and each item is rated for severity on a scale of $0-2(0=$ absent, $1=$ mild or intermittent, $2=$ severe $)$. The range of the total score is $0-38$. Higher scores are associated with more depressive symptoms. The scale has high inter-rater reliability and the Cronbach's alfa is 0.77 in this study.

To identify depressive symptoms, the DRS-RAI-HC was used. ${ }^{38-40}$ The DRS is an observational scale consisting of seven items that can be answered on a 3-point Likert scale, which varies from 0 (indicator not exhibited), 1 (indicator of this type exhibited at least once in the last 30 days and up to 5 days a week) or 2 (indicator exhibited daily or almost daily). The scores range from 0 to 14 , with higher scores indicating more depressive symptoms. The mood-items of the DRS-RAI-HC have good inter-rater reliability and the Cronbach's alfa is 0.83 in this study.
The GDS-15 was used as a self-report measure for depression. ${ }^{34,35}$ It comprises 15 questions about how the person with dementia has felt over the past week. Items require "yes/no" answers and the score range of the GDS-15 is 0 to 15 points, with higher scores indicating more depressive symptomatology. The Cronbach's alfa is 0.21 in this study. In the present study, the GDS-15 was administered to people with dementia with an MMSE-score of $\geq 15$ points in order to assess the depressive symptoms reliably.

The SF-36 was used to measure physical health. ${ }^{30,31}$ The caregiver completed this instrument. In this study, we used two of eight subscales: physical function (ten items) and physical role functioning (four items). Higher SF-36 scores indicated better health functioning. The two subscales have high internal consistency (Cronbach's alpha 0.92 for physical function and alpha 0.85 for physical role functioning in this study).

The SIP was used to measure physical health. ${ }^{32,33}$ The caregiver completed this instrument. In this study, we used two subscales: mobility range (ten items) and mobility control (12 items). Higher SIP scores indicated worse functioning. For both the mobility range and mobility control subscales the Cronbach's alpha is 0.77 in this study.

\section{Secondary outcomes}

The Dutch version of the Revised Memory and Behavior Problem Checklist (RMBPC) was used to assess the level of behavioral disturbance of the person with dementia. ${ }^{41,42}$ This is a 25 -item self-assessment questionnaire caregiver-report measure of observable behavioral problems in people with dementia (frequency scale). Caregivers were asked to rate the frequency of each problem on a 0 (never) to 4 (daily or more) 5-point Likert scale, with a higher score indicating the occurrence of a greater number of behavioral problems. The RMBPC is considered a reliable and valid tool for the empirical assessment of behavioral problems. The Cronbach's alfa is 0.84 in this study.

\section{Data analysis}

All the data were analyzed using SPSS Statistics 20 (IBM Corporation, Armonk, NY, USA). Firstly, descriptive statistics were calculated for participants in the intervention and comparison group and all the (outcome) variables were analyzed for differences between comparison and intervention group by means of independent-sample Student's $t$-tests for continuous variables and $\chi^{2}$ tests for categorical variables. Secondly, the baseline characteristics of dropouts and completers were compared using independent-sample $t$-tests and $\chi^{2}$ tests.

Thirdly, the effectiveness of the intervention on all outcomes was evaluated between the two groups by using 
generalized estimating equations (GEE). ${ }^{43-47}$ GEE are comparable with linear regression analysis with a correction for the dependency of the individual observations over time. For all GEE analyses, an exchangeable correlation matrix was used and all analyses were adjusted for the baseline value of the outcome. Characteristics of the people with dementia (age, sex, dementia type, day care, education level, GDS score, APOE- $\varepsilon 4$, baseline values for MMSE, comorbidity, and polypharmacy use) were identified a priori as potential covariates. We conducted a crude and an adjusted analysis to examine the effects over time. The crude analyses determined the effect of the intervention over time while controlling for the baseline values. The adjusted analyses determined the intervention effect over time while the covariates were added to the model. To evaluate the influence of the covariates on the intervention effect, effect modification was assessed by constructing interaction terms between the group and all covariates. In both GEE crude and adjusted analyses, firstly an overall intervention effect was estimated and secondly the intervention effect at 3 and 6 months follow-up. Furthermore, at all stages of data analysis, intention to treat (ITT) analyses were performed, including all participants as originally allocated after randomization. The logarithms of Cornell and DRS-RAI-HC were used, because of skewed data.

In addition to ITT analysis, homework-compliance analyses were performed. First, we performed the analysis only for dyads with full intervention compliance according to the per protocol principle. Only dyads who completed the entire intervention according to the protocol are counted toward the final results (completed 6-8 sessions, completed 30 minutes active exercise at least 3 days a week, and implemented pleasant activities in daily life for both the person with dementia and caregiver for at least 6 weeks according to protocol). Second, we performed the analysis for dyads with full compliance for exercise homework but not necessarily for full implementation of pleasant events in their daily life (completed 6-8 sessions and 30 minutes of active exercise for at least 3 days a week). Finally, we evaluated the outcomes of dyads with full compliance for planning pleasant activities only (implemented pleasant activities in daily life for both the person with dementia and the caregiver for at least 2 weeks). All dyads included in all the three compliance analyses completed a minimum of six of the eight home visits. For this additional compliance analysis, GEE were also used.

\section{Results}

\section{Enrollment and dropouts}

Figure 1 shows the participant flow. To detect an effect size of $d>0.40$ between the intervention and comparison groups with $\alpha=0.05$ and $\beta=0.80,78$ dyads in each group would have been needed, 156 dyads in total (100\%). Of the 146 dyads recruited for this study, in total 111 dyads fulfilled the inclusion criteria and were randomized to the intervention group $(\mathrm{n}=57)$ or comparison group $(\mathrm{n}=54)$ (Figure 1). In total, 98 (88\%) people with dementia completed post-measurement and $85(77 \%)$ people with dementia completed 6-month follow-up measurement. During the first 3 months, five people with dementia dropped out in the experimental group and eight in the comparison group. After 6 months, seven people with dementia dropped out in the experimental group and four in the comparison group. The reasons for dropping out were health problems, burden, death of the person with dementia, nursing home placement, and hospitalization. We found one significant result between people with dementia "dropouts" and people with dementia "completers". Compared to completers, dropouts had a significantly lower physical role functioning as measured with the SF-36.

\section{Sample characteristics}

At baseline, people with dementia were primarily male spouses (63\%) with dementia of the Alzheimer type (70\%). As shown in Table 1, the age ranged from 57 to 90 years ( mean $=77$; standard deviation SD $=7.458$ ), mean MMSE score was $21(\mathrm{SD}=5.191)$ and the mean Global Deterioration Score was $4.5(\mathrm{SD}=0.619)$. Their caregivers were primarily female spouses $(72 \%)$ with an age range from 35 to 92 years (mean $[\mathrm{M}]=72, \mathrm{SD}=10.09$ ). Their mean Epidemiologic Studies-Depression score at baseline was $10.93(\mathrm{SD}=7.70)$. No significant differences were found between the comparison and the intervention group at baseline in any of the sample characteristics and primary and secondary outcomes in persons with dementia or caregivers. Mean scores and SDs for both primary and secondary outcomes at different assessment points are presented in Table 2 .

\section{ITT analysis}

As shown in Table 3, GEE analysis among 111 dyads revealed no benefits of the multicomponent dyadic intervention on any of the outcomes. However, two significant results in favor of the comparison group were found in both crude and adjusted analysis. For DRS-RAI-HC we found a significant overall treatment effect (directed by a posttreatment effect) over time between the intervention and comparison group $(P<0.05)$ with higher depression scores for the intervention group. Furthermore, for the RMBPC, we found a significant overall effect over time between the intervention and comparison group $(P<0.05)$ with higher scores (more behavioral problems) for the intervention group. 


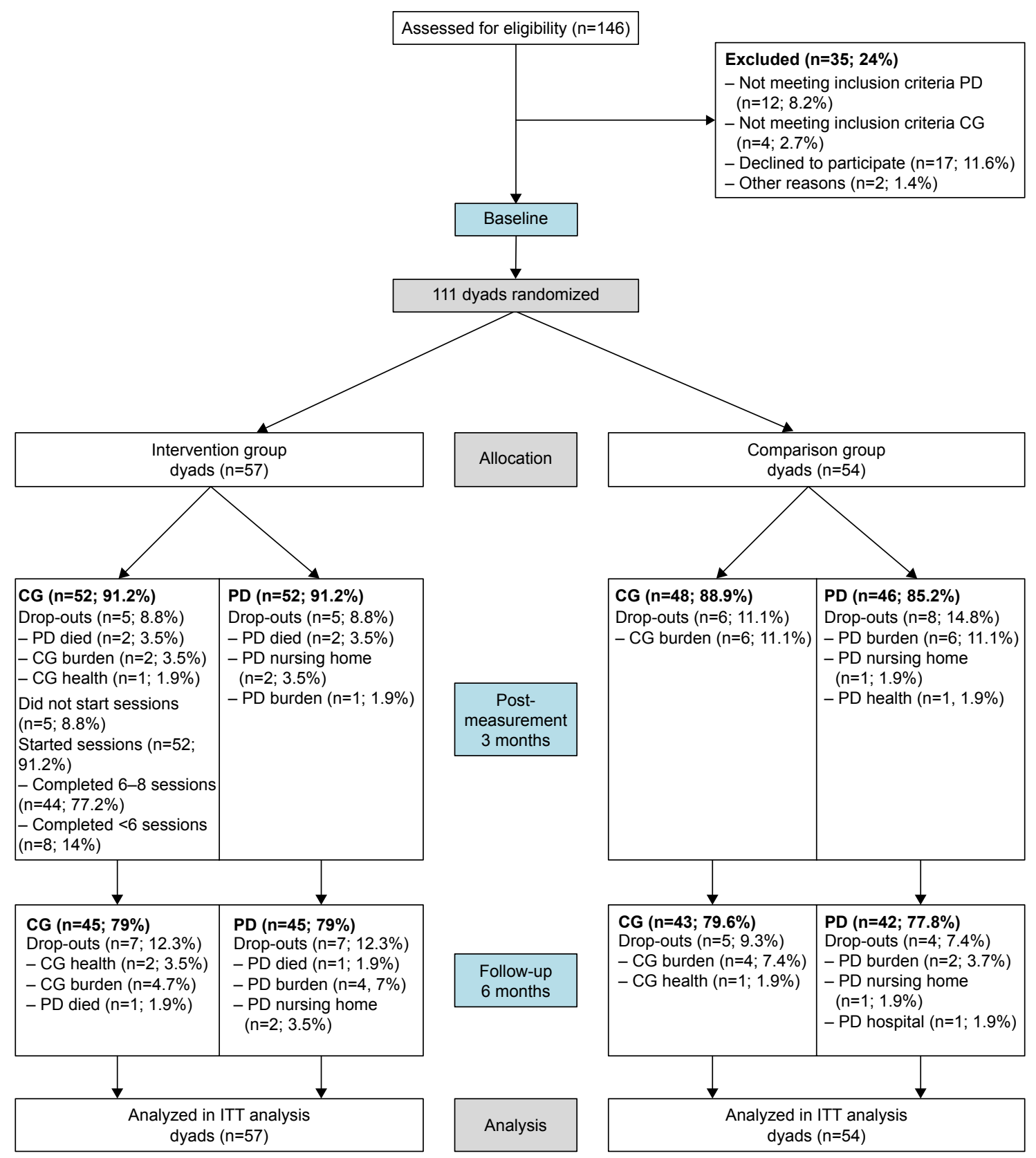

Figure I Flow chart of the progress of the present study performed in the Netherlands (2008-20I3).

Abbreviations: CG, caregiver; PD, person with dementia; ITT, intention to treat.

After adjusting for people with dementia characteristics, no significant changes were found.

\section{Compliance analyses}

As shown in Table 4, in the intervention group 44 dyads (77.2\%) completed all eight home visits. Five dyads (8.8\%) did not start with the home visits because of death or nursing home placement of the person with dementia. In the intervention group, 23 dyads (40.4\%) continued to exercise at home according to protocol (exercise compliance), and 18 dyads
(31.6\%) continued to plan pleasant activities according to protocol (pleasant activities compliance) after visits by their coach. Nine dyads were fully compliant with the intervention ([15.8\%] completed eight home visits, exercised three times a week, and planned pleasant activities according to protocol: so-called "per protocol analysis"). In total, three compliance analyses were carried out (full compliance [= per protocol analysis], exercise compliance, and pleasant activities compliance analysis): results from all three compliance analyses showed no benefits of the dyadic multicomponent intervention on any of 
Table I Baseline characteristics of people with dementia and their caregivers

\begin{tabular}{|c|c|c|c|c|}
\hline & $\begin{array}{l}\text { Total sample } \\
(\mathrm{N}=\text { III) }\end{array}$ & $\begin{array}{l}\text { Intervention } \\
(\mathrm{N}=57)\end{array}$ & $\begin{array}{l}\text { Comparison } \\
(\mathrm{N}=54)\end{array}$ & $\begin{array}{l}\text { P-value } \\
\text { (2-sided) }\end{array}$ \\
\hline \multicolumn{5}{|l|}{ People with dementia } \\
\hline Sex, n (\%) & & & & 0.05 \\
\hline Male & $70(63.1)$ & $31(54.4)$ & $39(72.2)$ & \\
\hline Female & $4 \mid(36.9)$ & $26(45.6)$ & I5 (27.8) & \\
\hline Dementia type, n (\%) & & & & 0.49 \\
\hline Alzheimer's & $78(70.3)$ & $42(73.7)$ & $36(66.7)$ & \\
\hline Vascular & $17(15.3)$ & $9(15.8)$ & $8(14.8)$ & \\
\hline Other & $16(14.4)$ & $6(10.5)$ & $10(18.5)$ & \\
\hline APOE-ع4 carrier, n (\%) & & & & 1 \\
\hline No & $53(47.7)$ & $27(50)$ & $26(48.1)$ & \\
\hline Yes & $55(49.5)$ & $27(50)$ & $28(51.9)$ & \\
\hline Day care, n (\%) & & & & 0.66 \\
\hline No & $57(5 \mathrm{I} .4)$ & $29(52.7)$ & $28(51.9)$ & \\
\hline I day & $7(6.3)$ & $5(9.1)$ & $2(3.7)$ & \\
\hline 2 days & $45(40.5)$ & $21(38.2)$ & $24(44.5)$ & \\
\hline Physical activity level during youth ( $<18$ years), n (\%) & & & & 0.87 \\
\hline No physical exercise & $17(15.6)$ & $9(15.8)$ & $8(15.4)$ & \\
\hline Physical exercise I-2 times a week & $31(28.4)$ & $15(26.3)$ & $16(30.8)$ & \\
\hline Physical exercise $\geq 3$ times a week & $61(56.0)$ & $33(57.9)$ & $28(53.8)$ & \\
\hline Physical activity level I 8-55 years, n (\%) & & & & 0.54 \\
\hline No physical exercise & $17(15.3)$ & II (19.3) & $6(11.5)$ & \\
\hline Physical exercise I-2 times a week & $34(30.6)$ & $17(29.8)$ & $17(32.7)$ & \\
\hline Physical exercise $\geq 3$ times a week & $58(52.3)$ & $29(50.9)$ & $29(55.8)$ & \\
\hline Physical activity level prior to trial, $\mathrm{n}(\%)$ & & & & 0.40 \\
\hline No physical exercise & $24(21.6)$ & $13(23.6)$ & II (20.8) & \\
\hline Physical exercise I-2 times a week & $46(41.4)$ & $26(47.3)$ & $20(37.7)$ & \\
\hline Physical exercise $\geq 3$ times a week & $38(34.2)$ & $16(29.1)$ & $22(4 \mid .5)$ & \\
\hline Age (range 57-90), mean (SD) & $77(7.5)$ & $76(7.6)$ & $78(7.2)$ & 0.1 \\
\hline Education (range 0-6), mean (SD) & $4.01(1.4)$ & $3.91(1.4)$ & $4.11(1.5)$ & 0.47 \\
\hline Comorbidity (range 0-5), mean (SD) & $1.65(1.3)$ & $1.68(1.2)$ & I.6I (I.3) & 0.76 \\
\hline Polypharmacy use (range 0-9), mean (SD) & $2.78(2.0)$ & $2.74(1.8)$ & $2.83(2.1)$ & 0.80 \\
\hline MMSE, mean (SD) & $21(5.2)$ & $21(4.9)$ & $21(5.6)$ & 0.91 \\
\hline Global Deterioration Scale, mean (SD) & $4.53(0.6)$ & $4.54(0.6)$ & $4.53(0.6)$ & 0.94 \\
\hline Geriatric Depression Scale, mean (SD) & $2.95(2.2)$ & $3.03(2.2)$ & $2.86(2.2)$ & 0.73 \\
\hline Cornell, mean (SD) & $6.20(4.6)$ & $6.32(4.2)$ & $5.85(5.1)$ & 0.7 \\
\hline DRS-RAI-HC, mean (SD) & $2.43(2.9)$ & $2.74(3.2)$ & $2.09(2.5)$ & 0.25 \\
\hline SF-36 physical function, mean (SD) & $60.72(28.9)$ & $58.77(28.6)$ & $64.58(28.5)$ & 0.31 \\
\hline SF-36 physical role function, mean (SD) & $44.82(41.4)$ & $41.67(41.0)$ & $48.15(42.0)$ & 0.85 \\
\hline SIP mobility control, mean (SD) & $24.77(21.8)$ & $26.67(22.8)$ & $22.22(20.9)$ & 0.29 \\
\hline SIP mobility range, mean (SD) & $38.83(25.8)$ & $35.96(23.7)$ & $41.89(27.9)$ & 0.23 \\
\hline RMBPC, mean (SD) & $28.22(10.4)$ & $28.39(10.1)$ & $28.04(10.7)$ & 0.86 \\
\hline \multicolumn{5}{|l|}{ Caregivers } \\
\hline Sex, n (\%) & & & & 0.19 \\
\hline Male & $31(27.9)$ & $19(33.3)$ & $12(22.2)$ & \\
\hline Female & $80(72.1)$ & $38(66.7)$ & $42(77.8)$ & \\
\hline Relationship, n (\%) & & & & 0.39 \\
\hline Spouse & $100(90.1)$ & $50(87.7)$ & $50(92.6)$ & \\
\hline Child and Other & II (9.9) & $7(12.3)$ & $4(7.4)$ & \\
\hline Age (range 35-92), mean (SD) & $72(10.1)$ & $73(9.9)$ & $71(10.31)$ & 0.47 \\
\hline Education (range 0-6), mean (SD) & $4.08(1.4)$ & $4.32(1.2)$ & $3.83(1.5)$ & 0.07 \\
\hline CES-D, mean (SD) & $10.93(7.7)$ & $10.84(6.9)$ & II.02 (8.6) & 0.91 \\
\hline
\end{tabular}

Abbreviations: SD, standard deviation; MMSE, Mini Mental State Examination; Cornell, Cornell Scale for Depression in Dementia; DRS-RAI-HC, Depression Rating Scale of the Resident Assessment Instrument Home Care; SF-36, Medical Outcome Study 36-item Short-Form Health Survey; SIP, Sickness Impact Profile; RMBPC, Revised Memory and Behavior Problem Checklist; CES-D, Centre for Epidemiologic Studies-Depression; APOE- 4 , apolipoprotein $\varepsilon 4$ allele. 
Table 2 Means (M) and standard deviations (SD) of the outcome variables at T0, TI, and T2 in ITT analysis

\begin{tabular}{|c|c|c|c|c|c|c|c|c|c|c|c|c|}
\hline & \multicolumn{6}{|c|}{$\begin{array}{l}\text { Intervention group } \\
(\mathrm{N}=57)\end{array}$} & \multicolumn{6}{|c|}{$\begin{array}{l}\text { Comparison group } \\
(\mathrm{N}=54)\end{array}$} \\
\hline & \multicolumn{2}{|c|}{$\begin{array}{l}\text { Baseline } \\
\text { (T0) }\end{array}$} & \multicolumn{2}{|c|}{$\begin{array}{l}\text { Post-measurement } \\
\text { (TI) }\end{array}$} & \multicolumn{2}{|c|}{$\begin{array}{l}6 \text { month } \\
\text { follow-up (T2) }\end{array}$} & \multicolumn{2}{|c|}{$\begin{array}{l}\text { Baseline } \\
\text { (T0) }\end{array}$} & \multicolumn{2}{|c|}{$\begin{array}{l}\text { Post-measurement } \\
\text { (TI) }\end{array}$} & \multicolumn{2}{|c|}{$\begin{array}{l}6 \text { month } \\
\text { follow-up (T2) }\end{array}$} \\
\hline & $M$ & SD & $M$ & SD & $\mathbf{M}$ & SD & M & SD & $M$ & SD & M & SD \\
\hline Cornell & 6.82 & 4.46 & 7.71 & 4.78 & 8.04 & 5.52 & 5.54 & 4.78 & 5.87 & 4.71 & 5.45 & 4.7I \\
\hline DRS-RAI-HC & 2.74 & 3.24 & 3.38 & 3.39 & 3.49 & 3.84 & 2.09 & 2.54 & 1.76 & 2.56 & 1.91 & 2.47 \\
\hline SF-36 physical function & 58.51 & 28.86 & 55.09 & 27.78 & 53.56 & 30.44 & 63.06 & 29.05 & 59.69 & 29.74 & 55.81 & 32.31 \\
\hline SF-36 physical role function & 41.67 & 41.01 & 24.03 & 34.29 & 23.33 & 32.16 & 48.15 & 42.02 & 29.69 & 35.60 & 36.05 & 39.83 \\
\hline SIP mobility control & 27.19 & 22.52 & 32.05 & 24.77 & 31.48 & 24.61 & 22.22 & 20.92 & 28.19 & 25.03 & 30.62 & 25.70 \\
\hline SIP mobility range & 35.96 & 23.74 & 42.88 & 29.33 & 49.33 & 29.34 & 41.85 & 27.61 & 43.40 & 28.99 & 48.60 & 27.82 \\
\hline RMBPC total & 28.39 & 10.12 & 30.87 & 12.54 & 33.49 & II.87 & 28.04 & $10.7 \mid$ & 26.32 & 10.01 & 27.36 & 10.89 \\
\hline
\end{tabular}

Abbreviations: Cornell, Cornell Scale for Depression in Dementia; DRS-RAI-HC, Depression Rating Scale of the Resident Assessment Instrument Home Care; SF-36, Medical Outcome Study 36-item Short-Form Health Survey; SIP, Sickness Impact Profile; RMBPC, Revised Memory and Behavior Problem Checklist; ITT, intention to treat; T0, baseline; TI, post-measurement; T2, 6 month follow-up.

the outcome measures of mood, behavior, and physical health in people with dementia assigned to the intervention group.

\section{Discussion}

In this RCT, we studied the effects of a multicomponent dyadic intervention study aimed at decreasing problems with the mood, behavior, and physical health of community dwelling people with dementia. The multicomponent intervention consisted of physical exercise training, psycho-education, communication skills training, and pleasant activities training. People with dementia were primarily male and diagnosed with Alzheimer's disease. Their family caregivers

Table 3 Intention to treat GEE intervention effects at baseline, 3, and 6 months on Cornell, DRS-RAI-HC, SIP subscales, SF-36 subscales, and RMBPC

GEE analysis - intention to treat*

$\mathrm{N}=$ III dyads (57 intervention group/54 comparison group)

\begin{tabular}{|c|c|c|c|c|c|}
\hline \multirow[t]{2}{*}{ Outcome measure } & & \multicolumn{2}{|l|}{ Crude model } & \multicolumn{2}{|l|}{ Adjusted model } \\
\hline & & $\beta(95 \% \mathrm{Cl})$ & $P$-value & $\beta(95 \% \mathrm{Cl})$ & $P$-value \\
\hline \multirow[t]{3}{*}{ Cornell } & Overall & $0.19(-0.06-0.43)$ & 0.13 & $0.17(-0.06-0.40)$ & 0.15 \\
\hline & $\mathrm{TI}$ & $0.17(-0.11-0.46)$ & 0.23 & $0.17(-0.07-0.40)$ & 0.16 \\
\hline & $\mathrm{T} 2$ & $0.20(-0.07-0.47)$ & 0.14 & $0.18(-0.05-0.41)$ & 0.13 \\
\hline \multirow[t]{3}{*}{ DRS-RAI-HC } & Overall & $0.29(0.11-0.46)$ & 0.02 & $0.31(0.14-0.48)$ & $<0.00$ \\
\hline & $\mathrm{TI}$ & $0.34(0.12-0.56)$ & $<0.00$ & $0.31(0.13-0.48)$ & $<0.00$ \\
\hline & $\mathrm{T} 2$ & $0.22(-0.00-0.45)$ & 0.05 & $0.32(0.14-0.49)$ & $<0.00$ \\
\hline SF-36 & Overall & $0.78(-5.23-6.79)$ & 0.80 & $2.24(-3.40-7.87)$ & 0.44 \\
\hline \multirow[t]{2}{*}{ Physical function } & $\mathrm{TI}$ & $-0.49(-6.69-5.71)$ & 0.88 & $2.30(-3.33-7.93)$ & 0.42 \\
\hline & $\mathrm{T} 2$ & $2.24(-6.23-10.72)$ & 0.60 & $2.19(-3.45-7.83)$ & 0.45 \\
\hline SF-36 & Overall & $-5.20(-|5.4|-5.0 \mid)$ & 0.32 & $-1.04(-|0.49-8.4|)$ & 0.83 \\
\hline \multirow[t]{2}{*}{ Physical role function } & $\mathrm{TI}$ & $-2.86(-14.88-9.16)$ & 0.64 & $-1.17(-10.60-8.23)$ & $0.8 \mathrm{I}$ \\
\hline & $\mathrm{T} 2$ & $-7.89(-21.44-5.66)$ & 0.25 & $-1.52(-10.9 \mid-7.87)$ & 0.75 \\
\hline SIP & Overall & $-2.10(-7.69-3.50)$ & 0.46 & $-0.62(-8.10-3.09)$ & 0.38 \\
\hline \multirow[t]{2}{*}{ Mobility control } & $\mathrm{TI}$ & $-0.60(-7.43-6.23)$ & 0.86 & $-0.48(-5.77-4.81)$ & 0.86 \\
\hline & $\mathrm{T} 2$ & $-3.81(-9.86-2.24)$ & 0.22 & $-0.62(-5.92-4.68)$ & 0.82 \\
\hline SIP & Overall & $4.57(-2.98-12.12)$ & 0.24 & $2.98(-4.68-10.64)$ & 0.45 \\
\hline \multirow[t]{2}{*}{ Mobility range } & $\mathrm{TI}$ & $4.41(-4.63-13.46)$ & 0.34 & $3.09(-4.57-10.74)$ & 0.43 \\
\hline & T2 & $5.07(-3.14-13.28)$ & 0.23 & $3.25(-4.39-10.89)$ & 0.40 \\
\hline \multirow[t]{3}{*}{ RMBPC } & Overall & $4.28(1.99-6.57)$ & $<0.00$ & $3.72(1.13-6.31)$ & 0.01 \\
\hline & $\mathrm{TI}$ & $3.77(1.32-6.23)$ & $<0.00$ & $3.75(1.16-6.34)$ & 0.01 \\
\hline & $\mathrm{T} 2$ & $4.94(2.12-7.77)$ & $<0.00$ & $3.82(1.25-6.39)$ & $<0.00$ \\
\hline
\end{tabular}

Notes: *Adjusted for baseline outcome; reference group is comparison group at all measurements; crude model: adjusted for baseline value of outcome measure; adjusted model: model further additionally adjusted for age, sex, education level, dementia type, APOE- $\varepsilon 4$, day care, Global Deterioration Scale score at T0, MMSE score at T0, comorbidity and, polypharmacy use; $\beta=$ regression coefficient. Because of skewed data we used logarithms of Cornell and DRS-RAI-HC; TI: post-measurement; T2: 6 month follow-up.

Abbreviations: GEE, generalized estimating equations; Cornell, Cornell Scale for Depression in Dementia; DRS-RAI-HC, Depression Rating Scale of the Resident Assessment Instrument Home Care; SF-36, Medical Outcome Study 36-item Short-Form Health Survey; SIP, Sickness Impact Profile; RMBPC, Revised Memory and Behavior Problem Checklist; $\mathrm{Cl}$, confidence interval; MMSE, Mini Mental State Examination; APOE- $\varepsilon 4$, apolipoprotein $\varepsilon 4$ allele. 
Table 4 Compliance to homework (exercise and pleasant activities planning) and completion of home visits of dyads assigned to intervention group $(\mathrm{N}=57)$

\begin{tabular}{|c|c|}
\hline Homework and home visits compliance $(\mathrm{N}=57)$ & n (\%) \\
\hline \multicolumn{2}{|l|}{ Home visits compliance (eight home visits) } \\
\hline Completed: $6-8$ home visits & $44(77.2)$ \\
\hline Partly completed: $<6$ home visits & $8(14)$ \\
\hline Not started with intervention: no home visits & $5(8.8)$ \\
\hline \multicolumn{2}{|l|}{ Homework exercise compliance (three times a week) } \\
\hline Exercise three or more times a week & $23(40.4)$ \\
\hline I-2 times weekly exercise & $16(28.1)$ \\
\hline 0 weekly exercise (intervention [partly] received) & $18(31.6)$ \\
\hline \multicolumn{2}{|l|}{ Planning pleasant activities compliance (without assistance from coach during at least 6 weeks) } \\
\hline Planned pleasant activities according to protocol: $>6$ weeks & $18(31.6)$ \\
\hline Partly planned pleasant activities: I- 6 weeks & $9(15.8)$ \\
\hline No pleasurable activities planned (intervention [partly] received) & $25(43.9)$ \\
\hline No pleasurable activities planned (not started with intervention) & $5(8.8)$ \\
\hline \multicolumn{2}{|l|}{ Combined homework and home visits compliance } \\
\hline Fully compliant according to protocol: completed eight home visits, exercised three times a week, and planned pleasant activities & $9(15.8)$ \\
\hline Moderate compliance & $43(75.4)$ \\
\hline Not started with intervention & $5(8.8)$ \\
\hline
\end{tabular}

were primarily female spouses. ITT GEE analyses showed no effects of the multicomponent dyadic intervention on the primary and secondary outcomes of people with dementia.

Many requirements for a high quality RCT were met. ${ }^{16}$ Randomization succeeded in showing that both groups were similar at baseline, and the dropout rates were reasonable for this vulnerable target group $(<30 \%) .{ }^{48}$ For people with dementia, the dropout rate in the study of Teri et $\mathrm{al}^{11}$ was similar at post-measurement. Evaluation of the data acquisition showed a careful collection of data with almost no missing data in the primary outcomes. Nevertheless, we did not find any beneficial impact of the intervention on any of the outcomes, including mood, behavior, and physical health; depression levels and behavioral problems even significantly increased in the active intervention group. A recent Cochrane review also revealed no evidence of benefit from exercise on depression, and furthermore, no effects on neuropsychiatric symptoms or cognition in people with dementia. ${ }^{49}$ Although, another recent review established that exercise training reduces depression levels in people with dementia, however, the effect of exercise on depression was small and its clinical relevance is unclear. ${ }^{50}$

The negative results in this study compared to the study of Teri et $\mathrm{al}^{11}$ might be explained by the translation, adaptation, and shortening of the intervention used in the US, and a different social context in both countries. In our adjusted and elaborated version of the intervention of Teri et al, ${ }^{11}$ we decided to concentrate on physical exercise, education, and a more expanded version of the pleasant activities training and to omit the time consuming $\mathrm{ABC}$ training. It is possible that cognitive reframing (based on $\mathrm{ABC}$ theory) was the active intervention component causing significant results in the study by Teri et al. ${ }^{11}$ Furthermore, our intervention consisted of eight home-based instruction sessions (instead of 12 instruction sessions in the study of Teri et al) ${ }^{11}$ in order to reduce the burden of a perceived lack of leisure time among the dyads who participated in our pilot study receiving the translated original intervention of Teri et al, and to comply with the Dutch health insurance regulations at the time of this study. Eight sessions was the maximum number of sessions that could be reimbursed at that time. Nevertheless, to help scientific design, we recommend that authors who are translating and adapting a comparable effective study for another region, adapt translation to local clinical practice and usual care in that specific region. Another explanation concerns the heterogeneous sample in terms of the subtype and severity of the disease and fitness in the present study. Teri et $\mathrm{al}^{11}$ included only people with Alzheimer's disease in their study, according to the National Institute of Neurological and Communicative Diseases and Related Disorders Association criteria for probable or possible Alzheimer's disease. ${ }^{51}$ A future consideration may be that studies should include a more homogeneous sample, but the authors of future studies should also be aware of the disadvantage of a more homogeneous sample: namely, much less generalizability of the study results. ${ }^{52} \mathrm{~A}$ final explanation of the difference in the results between the present study and Teri et al might be that compared with completers, dropouts had significantly lower SF-36 physical role functioning scores. A consequence might be that completers were "healthier", which may have resulted in less room for improvement. In Teri et al there were no significant demographic differences between 
those who completed the assessment at 3 months and those who did not. ${ }^{11}$

In addition, the negative results might be explained by a lack of room for improvement, and by experiencing the intervention as too much of a burden. In the Netherlands, for people with dementia living in the community, it is common to receive respite care in a day care facility for 1 or more days, where they participate in staffed activities such as music and exercise program. In our sample, half of the people with dementia received 1 or more days respite care in a day care facility. In addition to usual care, we examined the effectiveness of this intensive intervention. This could have hampered measuring the possible intervention effects in the case of improvement. In future studies, the impact of usual care provided in a specific country needs to be taken into account when the effectiveness of an intervention is being studied. ${ }^{16}$ In addition, dyads experienced the intervention as too confrontational. It could be that the multicomponent dyadic intervention raised awareness of the physical and mental capacities in persons with dementia. A few caregivers mentioned that doing physical exercises confronted the person with dementia with their inabilities, and some persons with dementia indicated that strengthening and balance exercises were too difficult. This might have hampered an improvement by the intervention in the intervention group and may even have caused increased depression and behavioral problem scores in people with dementia. In caregiver outcomes, we found comparable results: depression scores of caregivers appeared to rise over time in the intervention group, and caregiver health significantly improved in the comparison group. ${ }^{18}$ Furthermore, the physical exercise component was the most important named reason to participate in the study. The consequence was that we recruited already active dyads (almost $80 \%$ of the people with dementia were active alongside the intervention), which may have resulted in less room for improvement. ${ }^{18}$

Another explanation for the negative results might be that improving physical health might be only effective if the physical exercises are of moderate-to-high intensity, because physical exercise is correlated with brain volume, indicating a dose-response relationship..$^{53-55}$ The authors of a review studying the effects of physical activity on physical functioning and activities of daily life performance in elderly with dementia, found the largest improvements in interventions with the largest training volume: interventions with a duration of a minimum of 12 weeks, a frequency of three times a week, with 45-60-minute sessions. ${ }^{56}$ According to protocol, the goal of the present exercise component was to motivate dyads to complete active exercise at least 3 days a week during a minimum of 12 weeks. This duration (minimum of 12 weeks) and frequency (three times a week) is in accordance with Blankevoort et al. However, the "intensity" of exercise is not. Blankevoort et al advised to practice aerobic exercises for more than 45 minutes per session. ${ }^{56}$ However, there is still a lot to be said on the dosage (frequency, intensity, and duration) needed to elicit effects of exercise in people with dementia. ${ }^{57}$

In addition, during the 1-hour home visits, there was not enough time to practice intensive aerobic exercises like walking; of course the coaches stimulated aerobic exercises with the goal of increasing the time dyads walk by at least 20 minutes every day according to protocol. However, in terms of compliance, exercise homework was not performed as frequently as prescribed according to protocol in the present study: $68 \%$ of the dyads attempted their exercise homework (persons with dementia together with their caregiver completing 30 minutes of active exercise at least 3 days a week). Exercise treatment compliance in Teri et $\mathrm{al}^{11}$ showed that $91 \%$ of the dyads randomized to the intervention group attempted their exercise homework. In Teri et al, ${ }^{11}$ only $9 \%$ of the intervention group dyads did not complete any exercise homework in contrast to $32 \%$ of the dyads in our present study. Some caregivers indicated that following all the intervention components, including homework was too time consuming because of the time and energy needed to be invested, and too much of a burden, which affected homework adherence ${ }^{16}$ Feelings of burden among people with dementia due to the exercises might have been an important reason for not finding an improvement in mood, behavior, and physical health outcomes, even in participants with the highest level of adherence. More research is necessary to determine the right dose-response relationship between exercise and physical and mental health in people with dementia, and to identify barriers and facilitators to improving adherence.

In addition, for caregivers, it was difficult to motivate the person with dementia to exercise outside the home visits of the coach. Some caregivers indicated that remarks of the coach had more influence on the person with dementia than their own remarks in the absence of the coach, and that doing exercises confronted the person with dementia with diminished capacity resulting in demotivation. ${ }^{16}$ Motivation might be diminished in people with dementia regardless of their sex. Furthermore, we know that if exercise is not enjoyable it will not be sustained. ${ }^{22}$ To enhance the motivation for participation in the exercises, we tried to make the exercises extra attractive by the use of attributes like a ball and elastic and the use 
of music. In some cases, we did a little dancing in-between the exercises. For some people with dementia, and about half of the caregivers, the exercises with the ball they liked most, whereas other physical exercises were practiced less, or not practiced at all. ${ }^{16}$ Specific preferences of the persons with dementia may affectadherence to homework. Therefore, physical exercises should be tailored in accordance with participants' preferences and needs.$^{58}$ For future research the question arises how motivation in people with dementia can be enhanced, and also how to engineer and adapt exercises to fit with physical comorbidities, their personal situation, and needs and preferences. A recent systematic review presented different ways of modifying activities to enhance engagement in physical activities. One way presented to increase engagement is to match exercises to activities that are meaningful for the person with dementia. ${ }^{59}$

Some considerations concerning the study design need to be addressed. According to power calculation, the sample size was smaller than intended. In spite of tireless efforts, this was due to the difficulty in reaching participants. In the field of geriatric research, the present sample size is still reasonable. ${ }^{60}$ However, even with a much larger sample, we would not have been able to show benefits for the intervention group with differences in outcome found for both caregiver and people with dementia. In addition, the recruitment of participants for this study has generated a self-selected group of dyads who wanted "to stay active". For more than half of the dyads this was the most important reason to participate in the study. Since they were already active before joining the study, this might have resulted in a ceiling effect. Furthermore, injuries from adverse events or other temporary illnesses of the participants were not registered. Some participants and coaches mentioned that injuries or illness hampered the participants from executing the exercises at the prescribed intensity and appropriateness according to the protocol. Another consideration is that the study trial was not completely double-blind. Examiners were blinded to the group allocation and dyads were asked not to disclose their group allocation, although, in practice, group allocation became clear to most of the examiners. However, this had not positively skewed the results. Finally, we decided to exclude the GDS-15 from analysis because of a very low Cronbach's alfa indicating low scale reliability: Cronbach's alfa is 0.21 in this study. Although the GDS- 15 has been used in older people with dementia, some researchers have argued that it may not be the best way to assess symptoms of depression in dementia. ${ }^{61}$ Related to this issue, assessing the symptoms of depression in older people with dementia is difficult because of the overlap of symptoms of depression and dementia, such as difficulty concentrating and apathy. ${ }^{62}$ We have taken this into account during the design of the study: we measured depressive symptoms from different perspectives with subjective and more objective measures to create a complete overview of mood in people with dementia. Depressive symptoms of people with dementia were not only measured by clients' self-report (GDS-15), but also by caregivers' and clinical examiners' observation (Cornell and MDS-DRS-RAI-HC respectively, which are specifically designed for this particular population).

The primary outcomes for people with dementia were physical health, physical function, and mood. We hypothesized that people with dementia receiving the intervention would improve in these areas. Although no quantitative significant results were found on the primary outcomes, people with dementia participating in the intervention often commented positively about the pleasure they experienced and support they received. In additional qualitative research, people with dementia indicated, with regard to the physical exercise component, that they experienced pleasure, better mood, more self-esteem, increased awareness of the importance of exercise, and improvement in the quality of the relationship with their caregiver. ${ }^{16}$ These perceived benefits contrast with the lack of effectiveness of the intervention on used outcome measures. This highlights the need for measuring these specific outcomes to measure effectiveness of multicomponent dyadic interventions. We suppose that the working mechanism of the present multicomponent dyadic intervention, including physical exercise training supplemented with "duo" exercises for the person with dementia and the caregiver together, and a more expanded version of the pleasant activities training for both the person with dementia and the caregiver, is more directed at a better relationship and quality of life instead of primarily decreasing depression. For this reason, outcomes like relationship quality, pleasure, and self-esteem are more direct outcomes to measure the supposed effects of the present intervention. If these outcomes were quantitatively aligned to the intervention and assessed, the intervention might have resulted in changes on these variables and the intervention would have been quantitatively effective. For future research we recommend measuring these "experienced, positive" outcomes such as relationship quality with quantitative instruments alongside measuring negative feelings.

In conclusion, this high quality RCT showed no benefits of a multicomponent dyadic intervention comprised of physical exercise training, psycho-education, communication skills 
training, and pleasant activities training on mood, behavior, and physical health of community dwelling people with dementia. The negative results in this study compared to the study of Teri et al ${ }^{11}$ might be mainly explained by the translation, adaptation, and shortening of the intervention used in the US, and by a different social context in both countries. Dyads experienced the intervention as too much of a burden: outside the intervention, people with dementia received so much other health care (respite care and assistance of a case manager) and were already active. Furthermore, improving physical health might only be effective if the physical exercises are of moderate-to-high-intensity, and if physical exercises are tailored in accordance with participants' preferences and needs. In additional qualitative research, people with dementia participating in the intervention often commented positively about the pleasure they experienced and support they received. For future studies, it might be valuable to measure specific quality of life outcomes such as relationship quality, pleasure, and self-esteem in a dyadic focused intervention. All these aspects need to be taken into account, to improve future research on the effectiveness of home-based multicomponent dyadic interventions and to help people with dementia and their caregivers to continue their life at home.

\section{Acknowledgments}

We would like to thank the Dutch Health Insurers Innovation Foundation for their financial support. Furthermore, we thank all of the participants and research assistants who contributed to the study and the Mental Health research program of the EMGO Institute for Health and Care Research.

\section{Authors' contributions}

All authors contributed toward data analysis, drafting and revising the paper and agree to be accountable for all aspects of the work.

\section{Disclosure}

The authors report no conflicts of interest in this work.

\section{References}

1. Aalten P, de Vugt ME, Jaspers N, Jolles J, Verhey FR. The course of neuropsychiatric symptoms in dementia. Part I: findings from the two-year longitudinal Maasbed study. Int J Geriatr Psychiatry. 2005;20(6):523-530.

2. Lyketsos CG, Lopez O, Jones B, Fitzpatrick AL, Breitner J, DeKosky S. Prevalence of neuropsychiatric symptoms in dementia and mild cognitive impairment: results from the cardiovascular health study. JAMA. 2002;288(12):1475-1483.

3. Lyketsos CG, Steinberg M, Tschanz JT, Norton MC, Steffens DC, Breitner JC. Mental and behavioral disturbances in dementia: findings from the Cache County Study on Memory in Aging. Am J Psychiatry. 2000;157(5):708-714.
4. Yaffe K, Fox P, Newcomer R, et al. Patient and caregiver characteristics and nursing home placement in patients with dementia. JAMA. 2002;287(16):2090-2097.

5. Brodaty H, Arasaratnam C. Meta-analysis of nonpharmacological interventions for neuropsychiatric symptoms of dementia. Am J Psychiatry. 2012;169(9):946-953.

6. Acton GJ, Kang J. Interventions to reduce the burden of caregiving for an adult with dementia: a meta-analysis. Res Nurs Health. 2001; 24(5):349-360.

7. Olazarán J, Reisberg B, Clare L, et al. Nonpharmacological therapies in Alzheimer's disease: a systematic review of efficacy. Dement Geriatr Cogn Disord. 2010;30(2):161-178.

8. Brodaty H, Green A, Koschera A. Meta-analysis of psychosocial interventions for caregivers of people with dementia. J Am Geriatr Soc. 2003;51(5):657-664.

9. Van't Leven N, Prick A-E, Groenewoud JH, Roelofs PD, de Lange J, Pot AM. Dyadic interventions for community-dwelling people with dementia and their family caregivers: a systematic review. Int Psychogeriatr. 2013;25(10):1581-1603.

10. de Vugt ME, Stevens F, Aalten P, et al. Do caregiver management strategies influence patient behaviour in dementia? Int $J$ Geriatr Psychiatry. 2004;19(1):85-92.

11. Teri L, Gibbons LE, McCurry SM, et al. Exercise plus behavioral management in patients with Alzheimer disease. JAMA. 2003;290(15): 2015-2022.

12. Schulz R, O’Brien A, Czaja S, et al. Dementia caregiver intervention research In search of clinical significance. Gerontologist. 2002;42(5): 589-602.

13. Parker D, Mills S, Abbey J. Effectiveness of interventions that assist caregivers to support people with dementia living in the community: a systematic review. Int J Evid Based Health. 2008;6(2):137-172.

14. Brodaty H, Luscombe G. Psychological morbidity in caregivers is associated with depression in patients with dementia. Alzheimer Dis Assoc Disord. 1998;12(2):62-70.

15. Teri L, Truax P. Assessment of depression in dementia patients: association of caregiver mood with depression ratings. Gerontologist. 1994; 34(2):231-234.

16. Prick AE, de Lange J, van't Leven N, Pot AM. Process evaluation of a multicomponent dyadic intervention study with exercise and support for people with dementia and their family caregivers. Trials. 2014;15:401.

17. Prick AE, de Lange J, Scherder E, Pot AM. Home-based exercise and support programme for people with dementia and their caregivers: study protocol of a randomised controlled trial. BMC Public Health. 2011;11(1):894.

18. Prick AE, de Lange J, Twisk J, Pot AM. The effects of a multicomponent dyadic intervention on the psychological distress of family caregivers providing care to people with dementia: a randomized controlled trial. Int Psychogeriatr. 2015;27(12):2031-2044.

19. Teri L, McCurry SM, Buchner DM, et al. Exercise and activity level in Alzheimer's disease: a potential treatment focus. J Rehabil Res Dev. 1998;35(4):411-419.

20. Logsdon RG, McCurry SM, Teri L. A home health care approach to exercise for persons with Alzheimer's disease. Care Manag J. 2005; 6(2):90-97.

21. Vernooij-Dassen M, Draskovic I, McCleery J, Downs M. Cognitive reframing for carers of people with dementia. Cochrane Database Syst Rev. 2011;(11):CD005318.

22. Teri L, Logsdon RG, McCurry SM. Exercise interventions for dementia and cognitive impairment: the Seattle Protocols. J Nutr Health Aging. 2008;12(6):391-394.

23. Verhage F. [Intelligence and age: study with Dutch people aged 12-77]. Van Gorcum. 1964. Intelligentie en leeftijd bij volwassenen en bejaarden [Doctoral dissertation, University of Groningen]. Dutch.

24. Folstein MF, Folstein SE, McHugh PR. "Mini-mental state". A practical method for grading the cognitive state of patients for the clinician. J Psychiatr Res. 1975;12(3):189-198. 
25. Kok R, Verhey F. Dutch translation of the mini mental state examination (Folstein et al, 1975). 2002.

26. Reisberg B, Ferris SH, de Leon MJ, Crook T. The Global Deterioration Scale for assessment of primary degenerative dementia. $\mathrm{Am}$ J Psychiatry. 1982;139(9):1136-1139.

27. Foster PP, Rosenblatt KP, Kuljiš RO. Exercise-induced cognitive plasticity, implications for mild cognitive impairment and Alzheimer's disease. Front Neurol. 2011;2:28.

28. Nichol K, Deeny SP, Seif J, Camaclang K, Cotman CW. Exercise improves cognition and hippocampal plasticity in APOE epsilon4 mice. Alzheimers Dement. 2009;5(4):287-294.

29. Ilveskoski E, Lehtimäki T, Erkinjuntti T, Koivula T, Karhunen P. Rapid apolipoprotein E genotyping from mailed buccal swabs. J Neurosci Methods. 1998;79(1):5-8.

30. Van der Zee K, Sanderman R. RAND-36. Groningen: Northern Centre for Health Care Research, University of Groningen, the Netherlands; 1993:28.

31. Ware JE Jr, Sherbourne CD. The MOS 36-item short-form health survey (SF-36): I. Conceptual framework and item selection. Med Care 1992;30(6):473-483.

32. Bergner M, Bobbitt RA, Pollard WE, Martin DP, Gilson BS. The sickness impact profile: validation of a health status measure. Med Care. 1976;14(1):57-67.

33. Luttik A, Jacobs H, de Witte LP. Een Nederlandse versie van de Sickness Impact Profile [Dutch version of the Sickness Impact Profile]. Vakgroep Huisartsgeneeskunde, Rijksuniversiteit Utrecht; 1985. Dutch.

34. Sheikh JI, Yesavage JA. Geriatric Depression Scale (GDS): recent evidence and development of a shorter version. Clinical Gerontologist. 1986;5(1-2):165-173.

35. Kok R, Heeren TJ, Van Hemert A. The Geriatric Depression Scale. Tijdschrift Voor Psychiatrie. 1993;35(6):416-421.

36. Alexopoulos GS, Abrams RC, Young RC, Shamoian CA. Cornell Scale for Depression in Dementia. Biol Psychiatry. 1988;23(3):271-284.

37. Dröes R. Dutch translation of the Cornell Scale for Depression in Dementia. Amsterdam: VU University; 1993.

38. Frijters D, Achterberg W, Hirdes JP, Fries BE, Morris JN, Steel K. Geïntegreerd gezondheidsinformatiesysteem op basis van Resident Assessment Instrumenten. [Integrated health information system based on Resident Assessment Instruments]. Tijdschr Gerontol Geriatr. 2001;32(1):8-16. Dutch.

39. Morris JN, Fries BE, Bernabei R, et al. RAI-Home Care [RAI-HC] Assessment Manual for Version 2.0. Washington, DC: InterRAI Corporation; 1999

40. Morris JN, Fries BE, Steel K, et al. Comprehensive clinical assessment in community setting: applicability of the MDS-HC. J Am Geriatr Soc. 1997;45(8):1017-1024.

41. Teri L, Truax P, Logsdon R, Uomoto J, Zarit S, Vitaliano PP. Assessment of behavioral problems in dementia: the revised memory and behavior problems checklist. Psychol Aging. 1992;7(4):622-631.

42. Teunisse S, Haan R de, Walstra GJM, de Rooij SEJA, Zwart M. Behavioural problems in mild dementia: clinical relevance and methodological evaluation of the revised memory and behavioural problems checklist [PhD Thesis], the Netherlands: Universiteit van Amsterdam; 1997.

43. Liang KY, Zeger SL. Longitudinal data analysis using generalized linear models. Biometrika. 1986;73(1):13-22.

44. Zeger SL, Liang KY, Albert PS. Models for longitudinal data: a generalized estimating equation approach. Biometrics. 1988;44(4):1049-1060.

Clinical Interventions in Aging

\section{Publish your work in this journal}

Clinical Interventions in Aging is an international, peer-reviewed journal focusing on evidence-based reports on the value or lack thereof of treatments intended to prevent or delay the onset of maladaptive correlates of aging in human beings. This journal is indexed on PubMed Central, MedLine,
45. Twisk JW. Different statistical models to analyze epidemiological observational longitudinal data: an example from the Amsterdam Growth and Health Study. Int J Sports Med. 1997;18 Suppl 3:S216-224.

46. Twisk JW. Applied longitudinal data analysis for epidemiology: a practical guide. Cambridge University Press; 2013.

47. Twisk JW. Applied longitudinal data analysis for epidemiology: a practical guide. Cambridge University Press; 2003.

48. Bell ML, Kenward MG, Fairclough DL, Horton NJ. Differential dropout and bias in randomised controlled trials: when it matters and when it may not. BMJ. 2013;346:e8668.

49. Forbes D, Forbes SC, Blake CM, Thiessen EJ, Forbes S. Exercise programs for people with dementia. Cochrane Database Syst Rev. 2015;4: CD006489.

50. de Souto Barreto P, Demougeot L, Pillard F, Lapeyre-Mestre M, Rolland Y. Exercise training for managing behavioral and psychological symptoms in people with dementia: a systematic review and metaanalysis. Ageing Res Rev. 2015;24(Pt B):274-285.

51. McKhann G, Drachman D, Folstein M, Katzman R, Price D, Stadlan EM. Clinical diagnosis of Alzheimer's disease Report of the NINCDS-ADRDA Work Group under the auspices of Department of Health and Human Services Task Force on Alzheimer's Disease. Neurology. 1984;34(7):939-944.

52. Forbes D, Thiessen EJ, Blake CM, Forbes SC, Forbes S. Exercise programs for people with dementia. Cochrane Database Syst Rev. 2013; 12:CD006489.

53. Erickson KI, Kramer AF. Aerobic exercise effects on cognitive and neural plasticity in older adults. Br J Sports Med. 2009;43(1):22-24.

54. Vidoni ED, Honea RA, Billinger SA, Swerdlow RH, Burns JM. Cardiorespiratory fitness is associated with atrophy in Alzheimer's and aging over 2 years. Neurobiol Aging. 2012;33(8):1624-1632.

55. Burns JM, Cronk BB, Anderson HS, et al. Cardiorespiratory fitness and brain atrophy in early Alzheimer disease. Neurology. 2008;71(3): 210-216.

56. Blankevoort CG, van Heuvelen MJ, Boersma F, Luning H, de Jong J, Scherder EJ. Review of effects of physical activity on strength, balance, mobility and ADL performance in elderly subjects with dementia. Dement Geriatr Cogn Disord. 2010;30(5):392-402.

57. Phillips C, Baktir MA, Das D, Lin B, Salehi A. The Link Between Physical Activity and Cognitive Dysfunction in Alzheimer Disease. Phys Ther. 2015;95(7):1046-1060.

58. Thuné-Boyle IC, Iliffe S, Cerga-Pashoja A, Lowery D, Warner J. The effect of exercise on behavioral and psychological symptoms of dementia: towards a research agenda. Int Psychogeriatr. 2012;24(07): 1046-1057.

59. Trahan MA, Kuo J, Carlson MC, Gitlin LN. A systematic review of strategies to foster activity engagement in persons with dementia. Health Educ Behav. 2014;41(1 Suppl):70S-83S.

60. Armijo-Olivo S, Warren S, Magee D. Intention to treat analysis, compliance, drop-outs and how to deal with missing data in clinical research: a review. Physical Therapy Reviews. 2009;14(1):36-49.

61. Kørner A, Lauritzen L, Abelskov K, et al. The Geriatric depression Scale and the Cornell Scale for Depression in Dementia. A validity study. Nord J Psychiatry. 2006;60(5):360-364.

62. Downing LJ, Caprio TV, Lyness JM. Geriatric psychiatry review: differential diagnosis and treatment of the 3 D's-delirium, dementia, and depression. Current Psychiatry Reports. 2013;15(6):1-10.

CAS, Scopus and the Elsevier Bibliographic databases. The manuscript management system is completely online and includes a very quick and fair peer-review system, which is all easy to use. Visit http://www.dovepress. com/testimonials.php to read real quotes from published authors. 